\title{
Influence of variable viscosity on density-driven instabilities in capillary tubes
}

\author{
By M. PAYR, S. H. VANAPARTHY AND E. MEIBURG $\dagger$ \\ Department of Mechanical and Environmental Engineering, University of California, Santa \\ Barbara, CA 93106, USA
}

(Received 26 August 2003 and in revised form 24 September 2004)

\begin{abstract}
A linear stability analysis is conducted for the density-driven flow of variable viscosity miscible fluids in a vertically oriented capillary tube. The main goal is to assess the competition between the axisymmetric and the first azimuthal mode as a function of Rayleigh number, viscosity ratio and interfacial thickness parameter. In the absence of a net flow, the symmetry properties of the linearized set of equations indicate that the growth rates do not depend on which of the two fluids is the more viscous, although the shape of the eigenmodes does. For most parameter combinations, the first azimuthal mode is found to have larger growth rates than the axisymmetric mode. For thin interfaces and large Rayleigh numbers, however, the axisymmetric mode dominates above a certain viscosity ratio. An unexpected result is found regarding the influence of the interface thickness on the instability. For large viscosity ratios, intermediate interface thicknesses are found to be more unstable than either very thin or very thick interfaces. The reason for this behaviour is traced to a shift of the eigenfunctions towards the less viscous fluid, which allows the instability to grow in an overall less viscous environment. In the presence of a net axial flow, the upward and downward displacements of a more viscous fluid by a less viscous one are seen to result in the same growth rate. For large viscosity ratios, the axisymmetric mode becomes destabilized by the net flow, whereas the leading azimuthal mode is stabilized. This trend is in line with experimental observations.
\end{abstract}

\section{Introduction}

Vanaparthy, Meiburg \& Wilhelm (2003) present a linear stability analysis of the Rayleigh-Taylor instability in a vertically oriented capillary tube. Assuming constant viscosity fluids that are miscible with each other in all proportions, the authors determine dispersion relations and eigenmodes as functions of the Rayleigh number and the initial thickness of the interfacial region, based on the three-dimensional Stokes equations. For the case of a quiescent base state, the highest growth rates are invariably achieved by the three-dimensional mode with an azimuthal wavenumber of one. This is confirmed by related experiments by Kuang, Maxworthy \& Petitjeans (2004) for variable viscosity fluids. These experiments show that, in the absence of a net displacement, the interface between the two fluids initially develops an azimuthal instability. However, for long times and fully nonlinear fingers, the finger tip approaches the centre of the tube again. The authors subsequently demonstrate that in the presence of a small axial net flux the instability evolves in an axisymmetric

$\dagger$ Author to whom correspondence should be addressed. 


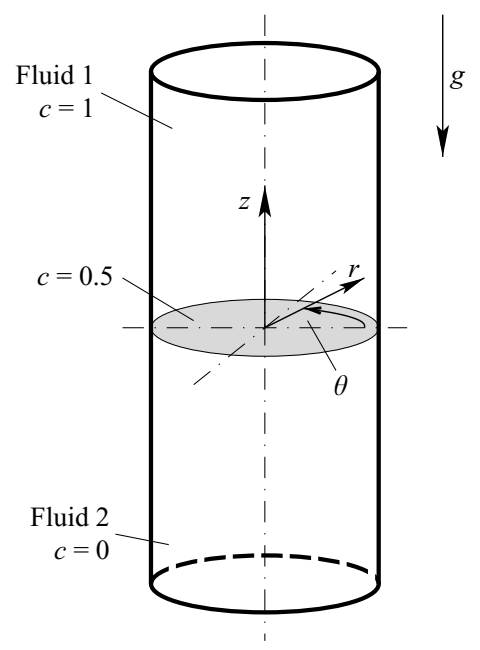

FIGURE 1. Sketch of the capillary tube and the initially flat interface at $z=0$. $c$ denotes the concentration of the heavier fluid.

fashion from the very beginning. They do not provide data regarding the minimum flow rate required to stabilize the azimuthal mode, and hence it is not clear whether or not this effect can be captured by linear stability theory. In order to assess this possibility, Vanaparthy et al. (2003) extend their constant viscosity linear stability analysis to cases involving a net axial flow, based on concentration profiles provided by Taylor (1953) for the dispersion of a passive scalar in a Poiseuille flow. However, for constant viscosity fluids, the results give no indication that the growth rate of the axisymmetric instability mode will overtake that of the leading azimuthal mode for the small net flow rates applied in the experiments. Hence, it appears possible that the experimental observations depend on the presence of viscosity variations, which may strongly affect the competition between axisymmetric and azimuthal modes. This is the question to be addressed in the following.

A literature review of density-driven instabilities in capillary tubes is provided by Vanaparthy et al. (2003) and will not be repeated here. Section 2 formulates the linear stability problem for variable viscosity miscible fluids and derives the corresponding generalized eigenvalue problem. The velocity and pressure approach for the threedimensional case is described along with its counterpart for axisymmetric perturbations, which is based on the vorticity and streamfunction variables. The corresponding numerical solution procedures are discussed in some detail. Results for quiescent base states are presented in $\S 3$, and compared with those for net axial flow in $\S 4$. Finally, $\S 5$ summarizes the findings and draws the main conclusions.

\section{Physical problem and governing equations}

We consider a vertically oriented capillary tube of diameter $d$, in which a heavier fluid 1 is placed above a lighter fluid 2 (figure 1). The fluids are miscible with each other in all proportions, with a constant diffusion coefficient $D$, and they have different viscosities. The linear stability analysis will be based on the standard incompressible conservation equations for mass, momentum, and species in cylindrical coordinates. Since typical flow velocities are small and a suitably defined Reynolds number is much smaller than $O(1)$, the convective terms in the momentum equations can safely 
be neglected, so that we employ the incompressible Stokes equations. We introduce characteristic scales for length $L^{*}$, viscosity $\mu^{*}$, velocity $U^{*}$, time $T^{*}$, pressure $P^{*}$ and density difference $R^{*}$ in the form

$$
\begin{aligned}
L^{*} & =d, \\
\mu^{*} & =\mu_{2}, \\
U^{*} & =\frac{\Delta \rho g d^{2}}{\mu_{2}}, \\
T^{*} & =\frac{\mu_{2}}{\Delta \rho g d}, \\
P^{*} & =\Delta \rho g d, \\
R^{*} & =\Delta \rho=\rho_{1}-\rho_{2} .
\end{aligned}
$$

Here, $\rho$ denotes the density and $g$ indicates the acceleration due to gravity, which is taken to point in the $-z$-direction. Note that from the linear stability equations to be derived below, it will become obvious that the growth rate does not depend on which of the two fluids is the more viscous. For this reason, we can limit ourselves, without loss of generality, to situations in which $\mu_{2}<\mu_{1}$, i.e. the heavier fluid is the more viscous. We thus obtain the following set of dimensionless equations

$$
\begin{gathered}
(\nabla \cdot v)=\frac{1}{r} \frac{\partial}{\partial r}\left(r v_{r}\right)+\frac{1}{r} \frac{\partial v_{\theta}}{\partial \theta}+\frac{\partial v_{z}}{\partial z}=0 \\
\frac{\partial p}{\partial r}=\frac{1}{r} \frac{\partial}{\partial r}\left(2 r \mu \frac{\partial v_{r}}{\partial r}\right)+\frac{1}{r} \frac{\partial}{\partial \theta}\left(\mu\left[r \frac{\partial}{\partial r}\left(\frac{v_{\theta}}{r}\right)+\frac{1}{r} \frac{\partial v_{r}}{\partial \theta}\right]\right)+\frac{\partial}{\partial z}\left(\mu\left[\frac{\partial v_{z}}{\partial r}+\frac{\partial v_{r}}{\partial z}\right]\right), \\
\frac{1}{r} \frac{\partial p}{\partial \theta}=\frac{1}{r^{2}} \frac{\partial}{\partial r}\left(r^{2} \mu\left[r \frac{\partial}{\partial r}\left(\frac{v_{\theta}}{r}\right)+\frac{1}{r} \frac{\partial v_{r}}{\partial \theta}\right]\right) \\
+\frac{1}{r} \frac{\partial}{\partial \theta}\left(2 \mu\left[\frac{1}{r} \frac{\partial v_{\theta}}{\partial \theta}+\frac{v_{r}}{r}\right]\right)+\frac{\partial}{\partial z}\left(\mu\left[\frac{\partial v_{\theta}}{\partial z}+\frac{1}{r} \frac{\partial v_{z}}{\partial \theta}\right]\right), \\
\frac{\partial p}{\partial z}=\frac{1}{r} \frac{\partial}{\partial r}\left(r \mu\left[\frac{\partial v_{z}}{\partial r}+\frac{\partial v_{r}}{\partial z}\right]\right)+\frac{1}{r} \frac{\partial}{\partial \theta}\left(\mu\left[\frac{\partial v_{\theta}}{\partial z}+\frac{1}{r} \frac{\partial v_{z}}{\partial \theta}\right]\right)+\frac{\partial}{\partial z}\left(2 \mu \frac{\partial v_{z}}{\partial z}\right)-c \\
\frac{\partial c}{\partial t}+\boldsymbol{v} \cdot \nabla c=\frac{1}{R a} \nabla^{2} c
\end{gathered}
$$

The dimensionless Rayleigh number, which indicates the ratio of convective to diffusive species transport, is defined as

$$
R a=\frac{\Delta \rho g d^{3}}{D \mu_{2}} .
$$

In order to close the above set of equations, we must specify density and viscosity as functions of the local concentration. In line with other authors, the density $\rho$ is assumed to be a linear function of $c$

$$
\rho=\rho_{2}+c\left(\rho_{1}-\rho_{2}\right)
$$

while the viscosity depends exponentially on the concentration

$$
\mu=\mu_{2} \mathrm{e}^{R c}, \quad R=\ln \frac{\mu_{1}}{\mu_{2}} .
$$




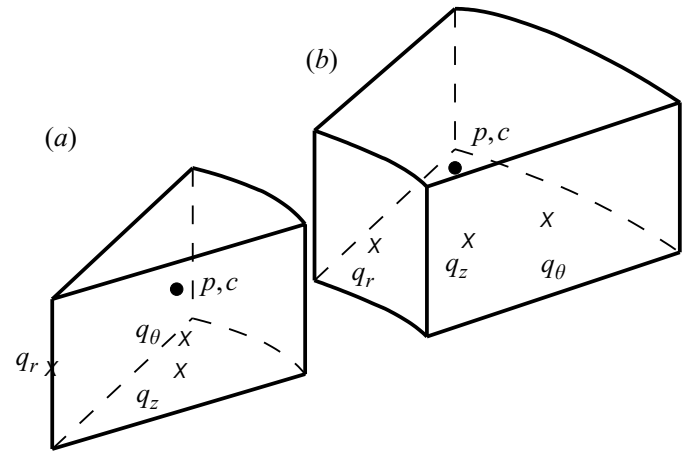

FIgURE 2. Sketch of the computational cells. (a) Cell at the axis. (b) Cell in the interior of the domain.

In order to avoid the appearance of singularities at $r=0$, Verzicco \& Orlandi (1996) recommend the use of a staggered grid. Here, the velocities are evaluated on the surface of the computational cell, whereas pressure and concentration are stored at its centre (figure 2). Furthermore, these authors propose to replace the velocity components $v_{r}$, $v_{\theta}$ and $v_{z}$ by the radial fluxes $q_{r}=v_{r} r, q_{\theta}=v_{\theta}$ and $q_{z}=v_{z}$, respectively. Thus, $q_{r}=0$ per definition on the axis, which avoids the problem of singularities. In terms of the new variables $q_{r}, q_{\theta}$ and $q_{z}$, the dimensionless continuity equation (2.7) becomes

$$
\frac{1}{r} \frac{\partial q_{r}}{\partial r}+\frac{1}{r} \frac{\partial q_{\theta}}{\partial \theta}+\frac{\partial q_{z}}{\partial z}=0
$$

The non-dimensional momentum equations (2.8)-(2.10) follow as

$$
\begin{aligned}
\frac{\partial p}{\partial r}= & \mathrm{e}^{R c}\left[-\frac{1}{r^{2}} \frac{\partial q_{r}}{\partial r}+\frac{1}{r} \frac{\partial^{2} q_{r}}{\partial r^{2}}+\frac{1}{r^{3}} \frac{\partial^{2} q_{r}}{\partial \theta^{2}}+\frac{1}{r} \frac{\partial^{2} q_{r}}{\partial z^{2}}-2 \frac{1}{r^{2}} \frac{\partial q_{\theta}}{\partial \theta}-2 R \frac{1}{r^{2}} \frac{\partial c}{\partial r} q_{r}-R \frac{1}{r^{2}} \frac{\partial c}{\partial \theta} q_{\theta}\right. \\
& \left.+2 R \frac{1}{r} \frac{\partial c}{\partial r} \frac{\partial q_{r}}{\partial r}+R \frac{1}{r} \frac{\partial c}{\partial \theta} \frac{\partial q_{\theta}}{\partial r}+R \frac{1}{r^{3}} \frac{\partial c}{\partial \theta} \frac{\partial q_{r}}{\partial \theta}+R \frac{\partial c}{\partial z} \frac{\partial q_{z}}{\partial r}+R \frac{1}{r} \frac{\partial c}{\partial z} \frac{\partial q_{r}}{\partial z}\right] \\
\frac{1}{r} \frac{\partial p}{\partial \theta}= & \mathrm{e}^{R c}\left[-\frac{1}{r^{2}} q_{\theta}+\frac{1}{r} \frac{\partial q_{\theta}}{\partial r}+\frac{\partial^{2} q_{\theta}}{\partial r^{2}}+\frac{1}{r^{2}} \frac{\partial^{2} q_{\theta}}{\partial \theta^{2}}+\frac{\partial^{2} q_{\theta}}{\partial z^{2}}+2 \frac{1}{r^{3}} \frac{\partial q_{r}}{\partial \theta}+2 R \frac{1}{r^{3}} \frac{\partial c}{\partial \theta} q_{r}-R \frac{1}{r} \frac{\partial c}{\partial r} q_{\theta}\right. \\
& \left.+R \frac{1}{r^{2}} \frac{\partial c}{\partial r} \frac{\partial q_{r}}{\partial \theta}+R \frac{\partial c}{\partial r} \frac{\partial q_{\theta}}{\partial r}+2 R \frac{1}{r^{2}} \frac{\partial c}{\partial \theta} \frac{\partial q_{\theta}}{\partial \theta}+R \frac{\partial c}{\partial z} \frac{\partial q_{\theta}}{\partial z}+R \frac{1}{r} \frac{\partial c}{\partial z} \frac{\partial q_{z}}{\partial \theta}\right] \\
\frac{\partial p}{\partial z}= & \mathrm{e}^{R c}\left[\frac{1}{r} \frac{\partial q_{z}}{\partial r}+\frac{\partial^{2} q_{z}}{\partial r^{2}}+\frac{1}{r^{2}} \frac{\partial^{2} q_{z}}{\partial \theta^{2}}+\frac{\partial^{2} q_{z}}{\partial z^{2}}+R \frac{1}{r} \frac{\partial c}{\partial r} \frac{\partial q_{r}}{\partial z}\right. \\
& \left.+R \frac{1}{r} \frac{\partial c}{\partial \theta} \frac{\partial q_{\theta}}{\partial z}+R \frac{\partial c}{\partial r} \frac{\partial q_{z}}{\partial r}+R \frac{1}{r^{2}} \frac{\partial c}{\partial \theta} \frac{\partial q_{z}}{\partial \theta}+2 R \frac{\partial c}{\partial z} \frac{\partial q_{z}}{\partial z}\right]-c
\end{aligned}
$$

and the concentration equation (2.11) takes the form

$$
\frac{\partial c}{\partial t}+\frac{1}{r} q_{r} \frac{\partial c}{\partial r}+\frac{1}{r} q_{\theta} \frac{\partial c}{\partial \theta}+q_{z} \frac{\partial c}{\partial z}=\frac{1}{R a}\left[\frac{\partial^{2} c}{\partial r^{2}}+\frac{1}{r} \frac{\partial c}{\partial r}+\frac{1}{r^{2}} \frac{\partial^{2} c}{\partial \theta^{2}}+\frac{\partial^{2} c}{\partial z^{2}}\right] .
$$

\subsection{Linearization}

Initially, we will consider base states in which the fluids are at rest, and the initial concentration profile is defined by an error function of the form

$$
\bar{c}=\frac{1}{2}+\frac{1}{2} \operatorname{erf}\left(\frac{z}{\delta}\right)
$$




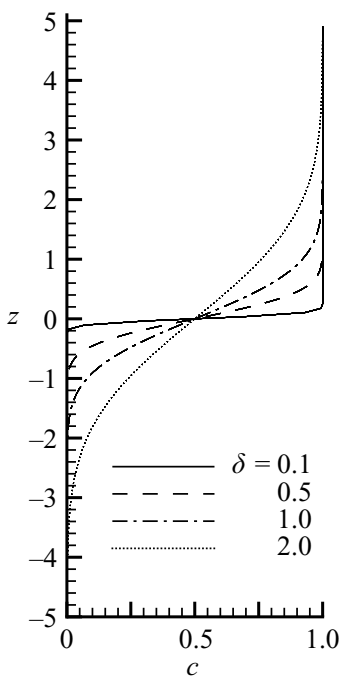

FIGURE 3. Base concentration profile $\bar{c}(z)$ for selected values of the interfacial thickness parameter $\delta$.

Here, $\delta$ represents the interface thickness (figure 3 ). Later on, we will also address base states characterized by steady purely axial flows whose exact form will be discussed at that time. For this reason, the linear stability equations will be derived for the case of a purely axial base flow. Each variable is decomposed into its base state $\left(^{-}\right)$and perturbation (') components

$$
\left(\begin{array}{c}
q_{r} \\
q_{\theta} \\
q_{z} \\
p \\
c
\end{array}\right)(r, \theta, z, t)=\left(\begin{array}{c}
0 \\
0 \\
\bar{q}_{z}(r) \\
\bar{p}(r, z) \\
\bar{c}(r, z)
\end{array}\right)+\left(\begin{array}{c}
q_{r}^{\prime} \\
q_{\theta}^{\prime} \\
q_{z}^{\prime} \\
p^{\prime} \\
c^{\prime}
\end{array}\right)(r, \theta, z, t)
$$

The perturbations are assumed to be wavelike in the circumferential direction

$$
\left(\begin{array}{l}
q_{r}^{\prime} \\
q_{\theta}^{\prime} \\
q_{z}^{\prime} \\
p^{\prime} \\
c^{\prime}
\end{array}\right)(r, \theta, z, t)=\left(\begin{array}{c}
\hat{q}_{r}(r, z) \cos (\beta \theta) \\
\hat{q}_{\theta}(r, z) \sin (\beta \theta) \\
\hat{q}_{z}(r, z) \cos (\beta \theta) \\
\hat{p}(r, z) \cos (\beta \theta) \\
\hat{c}(r, z) \cos (\beta \theta)
\end{array}\right) \mathrm{e}^{\sigma t} .
$$

The quantities with circumflexes denote the two-dimensional eigenfunctions. Note that only integral wavenumbers $\beta$ have physical meaning. By substituting the decomposition defined in (2.21) into the dimensionless equations (2.15)-(2.19), subtracting out the base-state equations, and neglecting all terms of higher order in the perturbations, the system of equations takes the form

$$
\begin{gathered}
\frac{\partial \hat{q}_{r}}{\partial r}+\beta \hat{q}_{\theta}+r \frac{\partial \hat{q}_{z}}{\partial z}=0 \\
\frac{\partial \hat{p}}{\partial r}=\mathrm{e}^{R \bar{c}}\left[-\frac{1}{r^{2}} \frac{\partial \hat{q}_{r}}{\partial r}+\frac{1}{r} \frac{\partial^{2} \hat{q}_{r}}{\partial r^{2}}-\frac{1}{r^{3}} \beta^{2} \hat{q}_{r}+\frac{1}{r} \frac{\partial^{2} \hat{q}_{r}}{\partial z^{2}}-2 \frac{1}{r^{2}} \beta \hat{q}_{\theta}-2 R \frac{1}{r^{2}} \frac{\partial \bar{c}}{\partial r} \hat{q}_{r}\right. \\
\left.+2 R \frac{1}{r} \frac{\partial \bar{c}}{\partial r} \frac{\partial \hat{q}_{r}}{\partial r}+R \frac{\partial \bar{c}}{\partial z} \frac{\partial \hat{q}_{z}}{\partial r}+R \frac{\partial \bar{q}_{z}}{\partial r} \frac{\partial \hat{c}}{\partial z}+R^{2} \frac{\partial \bar{c}}{\partial z} \frac{\partial \bar{q}_{z}}{\partial r} \hat{c}+R \frac{1}{r} \frac{\partial \bar{c}}{\partial z} \frac{\partial \hat{q}_{r}}{\partial z}\right]
\end{gathered}
$$




$$
\begin{aligned}
-\frac{1}{r} \beta \hat{p}= & \mathrm{e}^{R \bar{c}}\left[-\frac{1}{r^{2}} \hat{q}_{\theta}+\frac{1}{r} \frac{\partial \hat{q}_{\theta}}{\partial r}+\frac{\partial^{2} \hat{q}_{\theta}}{\partial r^{2}}-\frac{1}{r^{2}} \beta^{2} \hat{q}_{\theta}+\frac{\partial^{2} \hat{q}_{\theta}}{\partial z^{2}}-2 \frac{1}{r^{3}} \beta \hat{q}_{r}\right. \\
& \left.-R \frac{1}{r} \frac{\partial \bar{c}}{\partial r} \hat{q}_{\theta}-R \frac{1}{r^{2}} \beta \frac{\partial \bar{c}}{\partial r} \hat{q}_{r}+R \frac{\partial \bar{c}}{\partial r} \frac{\partial \hat{q}_{\theta}}{\partial r}+R \frac{\partial \bar{c}}{\partial z} \frac{\partial \hat{q}_{\theta}}{\partial z}-R \frac{1}{r} \beta \frac{\partial \bar{c}}{\partial z} \hat{q}_{z}\right] \\
\frac{\partial \hat{p}}{\partial z}= & \mathrm{e}^{R \bar{c}}\left[\frac{1}{r} \frac{\partial \hat{q}_{z}}{\partial r}+\frac{\partial^{2} \hat{q}_{z}}{\partial r^{2}}-\frac{1}{r^{2}} \beta^{2} \hat{q}_{z}+\frac{\partial^{2} \hat{q}_{z}}{\partial z^{2}}+R \frac{1}{r} \frac{\partial \bar{c}}{\partial r} \frac{\partial \hat{q}_{r}}{\partial z}+R \frac{\partial \bar{c}}{\partial r} \frac{\partial \hat{q}_{z}}{\partial r}+R \frac{1}{r} \frac{\partial \bar{q}_{z}}{\partial r} \hat{c}\right. \\
& \left.+R \frac{\partial^{2} \bar{q}_{z}}{\partial r^{2}} \hat{c}+R \frac{\partial \bar{q}_{z}}{\partial r} \frac{\partial \hat{c}}{\partial r}+2 R \frac{\partial \bar{c}}{\partial z} \frac{\partial \hat{q}_{z}}{\partial z}+R^{2} \frac{\partial \bar{c}}{\partial r} \frac{\partial \bar{q}_{z}}{\partial r} \hat{c}\right]-\hat{c} \\
\sigma \hat{c}+ & \frac{1}{r} \frac{\partial \bar{c}}{\partial r} \hat{q}_{r}+\bar{q}_{z} \frac{\partial \hat{c}}{\partial z}+\frac{\partial \bar{c}}{\partial z} \hat{q}_{z}=\frac{1}{R a}\left[\frac{\partial^{2} \hat{c}}{\partial r^{2}}+\frac{1}{r} \frac{\partial \hat{c}}{\partial r}-\frac{1}{r^{2}} \beta^{2} \hat{c}+\frac{\partial^{2} \hat{c}}{\partial z^{2}}\right]
\end{aligned}
$$

\subsection{Three-dimensional perturbations}

The above system of linear equations can be written in matrix form as

$$
\boldsymbol{A} \phi=\sigma \boldsymbol{B} \phi
$$

which represents a generalized eigenvalue problem with the eigenvector

$$
\phi=\left(\hat{p}, \hat{q}_{r}, \hat{q}_{\theta}, \hat{q}_{z}, \hat{c}\right)^{T}
$$

and $\sigma$ as its eigenvalue. The form of the matrices $\boldsymbol{A}$ and $\boldsymbol{B}$ is provided by the governing equations $(2.23)-(2.27)$ as

with

$$
\boldsymbol{A}=\left(\begin{array}{ccccc}
\mathbf{0} & \frac{\partial}{\partial r} & \beta & r \frac{\partial}{\partial z} & \mathbf{0} \\
-\mathrm{e}^{-R \bar{c}} \frac{\partial}{\partial r} & \boldsymbol{M}_{\mathbf{1}} & -\frac{2 \beta}{r^{2}} & R \frac{\partial \bar{c}}{\partial z} \frac{\partial}{\partial r} & \boldsymbol{M}_{\mathbf{5}} \\
\mathrm{e}^{-R \bar{c} \frac{\beta}{r}} & \boldsymbol{M}_{\mathbf{6}} & \boldsymbol{M}_{\mathbf{2}} & -R \frac{\beta}{r} \frac{\partial \bar{c}}{\partial z} & \mathbf{0} \\
-\mathrm{e}^{-R \bar{c}} \frac{\partial}{\partial z} & R \frac{1}{r} \frac{\partial \bar{c}}{\partial r} \frac{\partial}{\partial z} & \mathbf{0} & \boldsymbol{M}_{\mathbf{3}} & \boldsymbol{M}_{\mathbf{7}} \\
\mathbf{0} & -\frac{1}{r} \frac{\partial \bar{c}}{\partial r} & \mathbf{0} & -\frac{\partial \bar{c}}{\partial z} & \boldsymbol{M}_{\mathbf{4}}
\end{array}\right),
$$

$$
\begin{aligned}
& \boldsymbol{M}_{\mathbf{1}}=-\frac{1}{r^{2}} \frac{\partial}{\partial r}+\frac{1}{r} \frac{\partial^{2}}{\partial r^{2}}-\frac{1}{r^{3}} \beta^{2}+\frac{1}{r} \frac{\partial^{2}}{\partial z^{2}}-2 R \frac{1}{r^{2}} \frac{\partial \bar{c}}{\partial r}+2 R \frac{1}{r} \frac{\partial \bar{c}}{\partial r} \frac{\partial}{\partial r}+R \frac{1}{r} \frac{\partial \bar{c}}{\partial z} \frac{\partial}{\partial z} \\
& \boldsymbol{M}_{\mathbf{2}}=-\frac{1}{r^{2}}+\frac{1}{r} \frac{\partial}{\partial r}+\frac{\partial^{2}}{\partial r^{2}}-\frac{1}{r^{2}} \beta^{2}+\frac{\partial^{2}}{\partial z^{2}}-R \frac{1}{r} \frac{\partial \bar{c}}{\partial r}+R \frac{\partial \bar{c}}{\partial r} \frac{\partial}{\partial r}+R \frac{\partial \bar{c}}{\partial z} \frac{\partial}{\partial z} \\
& \boldsymbol{M}_{\mathbf{3}}=\frac{1}{r} \frac{\partial}{\partial r}+\frac{\partial^{2}}{\partial r^{2}}-\frac{1}{r^{2}} \beta^{2}+\frac{\partial^{2}}{\partial z^{2}}+R \frac{\partial \bar{c}}{\partial r} \frac{\partial}{\partial r}+2 R \frac{\partial \bar{c}}{\partial z} \frac{\partial}{\partial z} \\
& \boldsymbol{M}_{4}=\frac{1}{R a}\left[\frac{1}{r} \frac{\partial}{\partial r}+\frac{\partial^{2}}{\partial r^{2}}-\frac{1}{r^{2}} \beta^{2}+\frac{\partial^{2}}{\partial z^{2}}\right]-\bar{q}_{z} \frac{\partial}{\partial z}, \\
& \boldsymbol{M}_{5}=R \frac{\partial \bar{q}_{z}}{\partial r} \frac{\partial}{\partial z}+R^{2} \frac{\partial \bar{c}}{\partial z} \frac{\partial \bar{q}_{z}}{\partial r}, \\
& \boldsymbol{M}_{\mathbf{6}}=-\frac{2 \beta}{r^{3}}-R \frac{1}{r^{2}} \beta \frac{\partial \bar{c}}{\partial r}, \\
& \boldsymbol{M}_{7}=R \frac{1}{r} \frac{\partial \bar{q}_{z}}{\partial r}+R \frac{\partial^{2} \bar{q} z}{\partial r^{2}}+R \frac{\partial \bar{q}_{z}}{\partial r} \frac{\partial}{\partial r}+R^{2} \frac{\partial \bar{c}}{\partial r} \frac{\partial \bar{q}_{z}}{\partial r}-\mathrm{e}^{-R \bar{c}} .
\end{aligned}
$$


The numerical implementation follows the approach outlined by Vanaparthy et al. (2003) for the constant viscosity case. The equations are discretized on a domain that extends from the axis $(r=0)$ to the outer wall $(r=0.5)$ in the $r$-direction, and from $-l / 2$ to $l / 2$ in the $z$-direction. Second-order finite differencing is applied in both directions. In the radial direction, the nodes are spaced equidistantly. To resolve the strong axial gradients in the interfacial region, we use a stretched grid on the two subdomains $z \geqslant 0$ and $z \leqslant 0$ that concentrates the grid points near $z=0$. For this purpose, a mapping function provided by Fletcher (1991) is employed

$$
z_{i}=s_{i} \frac{l}{2},
$$

where

$$
s_{i}=P \eta_{i}+(1-P)\left(1-\frac{\tanh \left[Q\left(1-\eta_{i}\right)\right]}{\tanh Q}\right),
$$

with

$$
\eta_{i}=\frac{i-1}{n-1} .
$$

Here, $n$ represents the number of axial points within each subdomain, while $P$ and $Q$ are parameters to be chosen appropriately, in order to obtain a suitable distribution of grid points.

At the wall of the tube, all velocity components are assumed to vanish, along with the normal derivative of the concentration perturbation. The vertical domain boundaries are sufficiently far away from the interface, so that homogeneous Dirichlet conditions for all velocity components, as well as the concentration perturbation, can be prescribed. For the pressure variable, no boundary conditions are necessary, owing to the staggered grid. To keep the computational effort in an acceptable range, the numerical eigenvalue problem is solved iteratively for the leading eigenvalues by an Arnoldi method (Sorensen 1992). For the computational implementation, we use the public domain software package ARPACK (Maschhoff \& Sorensen 1996). Test calculations and comparisons with direct solvers for a system of linear equations (LAPACK) indicate that in this way the eigenvalues can be computed to a high degree of accuracy. However, at high $R a$ numbers, the corresponding eigenfunctions are not always fully converged, and the overall rate of convergence is slow. Consequently, we follow the suggestion by Graf, Meiburg \& Härtel (2002), which is also used by Vanaparthy et al. (2003), and slightly modify the original system of equations by adding a temporal derivative to the momentum equations. This results in the addition of a Reynolds-number-like parameter $R e$ to the corresponding positions on the main diagonal of matrix B. According to Vanaparthy et al. (2003), a value of $R e=0.001$ accelerates the convergence considerably, while having a negligible influence on the magnitude of the eigenvalue.

The domain length has to be chosen carefully, especially for small values of $\beta$, since it strongly influences some of the elements of $\boldsymbol{A}$. Several test calculations were performed in order to determine the optimal choice of domain length $l$ as a function of circumferential wavenumber $\beta$ and interface thickness $\delta$. Table 1 demonstrates the computational requirements for $\delta \leqslant 1$. For $\delta=2$, the minimal domain length is $l=10$. The value of each parameter was determined by means of a sensitivity analysis, in order to keep the error in the eigenvalue below $0.5 \%$. For the largest calculation, the resulting matrix $\boldsymbol{A}$ has a size of $5 N_{r} N_{z} \times 5 N_{r} N_{z}=11495 \times 11495$ elements. 


$\begin{array}{lcccrrr}\beta & 0.1 & 0.25 & 0.5 & 1 & 2 & 3 \\ l & 20 & 10 & 5 & 5 & 5 & 5 \\ N_{z} & 121 & 81 & 61 & 61 & 61 & 61 \\ N_{r} & 19 & 19 & 17 & 15 & 15 & 15\end{array}$

TABLE 1. Computational parameters for $\delta \leqslant 1$ and the case of three-dimensional perturbations.

\subsection{Axisymmetric perturbations}

For axisymmetric perturbations, it is convenient to write the governing equations in terms of the streamfunction $(\psi)$ and vorticity $(\omega)$ variables

$$
v_{r}=-\frac{1}{r} \frac{\partial \psi}{\partial z}, \quad v_{z}=\frac{1}{r} \frac{\partial \psi}{\partial r}, \quad \omega=\frac{\partial v_{r}}{\partial z}-\frac{\partial v_{z}}{\partial r} .
$$

By proceeding analogously to the case of three-dimensional perturbations, we obtain a generalized eigenvalue problem of the form

$$
\left(\begin{array}{ccc}
\boldsymbol{M}_{1} & \boldsymbol{I} & \mathbf{0} \\
\boldsymbol{M}_{4} & \boldsymbol{M}_{2} & \boldsymbol{M}_{5} \\
\boldsymbol{M}_{6} & \mathbf{0} & \boldsymbol{M}_{3}
\end{array}\right)\left(\begin{array}{l}
\hat{\psi} \\
\hat{\omega} \\
\hat{c}
\end{array}\right)=\sigma\left(\begin{array}{lll}
\mathbf{0} & \mathbf{0} & \mathbf{0} \\
\mathbf{0} & \mathbf{0} & \mathbf{0} \\
\mathbf{0} & \mathbf{0} & \boldsymbol{I}
\end{array}\right)\left(\begin{array}{l}
\hat{\psi} \\
\hat{\omega} \\
\hat{c}
\end{array}\right),
$$

with

$$
\begin{aligned}
\boldsymbol{M}_{\mathbf{1}}= & \frac{1}{r} \frac{\partial^{2}}{\partial z^{2}}+\frac{1}{r} \frac{\partial^{2}}{\partial r^{2}}-\frac{1}{r^{2}} \frac{\partial}{\partial r}, \\
\boldsymbol{M}_{\mathbf{2}}= & \frac{1}{r} \frac{\partial}{\partial r}+\frac{\partial^{2}}{\partial r^{2}}+\frac{\partial^{2}}{\partial z^{2}}-\frac{1}{r^{2}}+2 R \frac{\partial \bar{c}}{\partial z} \frac{\partial}{\partial z}+R^{2}\left(\frac{\partial \bar{c}}{\partial z}\right)^{2} \\
& +R \frac{\partial^{2} \bar{c}}{\partial z^{2}}+R \frac{\partial \bar{c}}{\partial r} \frac{\partial}{\partial r}-R^{2}\left(\frac{\partial \bar{c}}{\partial r}\right)^{2}-R \frac{\partial^{2} \bar{c}}{\partial r^{2}}, \\
\boldsymbol{M}_{\mathbf{3}}= & \frac{1}{R a}\left[\frac{\partial^{2}}{\partial r^{2}}+\frac{1}{r} \frac{\partial}{\partial r}+\frac{\partial^{2}}{\partial z^{2}}\right]-\bar{q}_{z} \frac{\partial}{\partial z}, \\
\boldsymbol{M}_{\mathbf{4}}= & -2 R^{2} \frac{1}{r^{2}}\left(\frac{\partial \bar{c}}{\partial z}\right)^{2} \frac{\partial}{\partial r}-2 R \frac{1}{r^{2}} \frac{\partial^{2} \bar{c}}{\partial z^{2}} \frac{\partial}{\partial r}-2 R \frac{1}{r} \frac{\partial \bar{c}}{\partial z} \frac{\partial^{3}}{\partial r^{2} \partial z} \\
& +2 R^{2} \frac{1}{r^{2}}\left(\frac{\partial \bar{c}}{\partial r}\right)^{2} \frac{\partial}{\partial r}+2 R \frac{1}{r^{2}} \frac{\partial^{2} \bar{c}}{\partial r^{2}} \frac{\partial}{\partial r}+2 R^{2} \frac{1}{r^{2}} \frac{\partial \bar{c}}{\partial r} \frac{\partial \bar{c}}{\partial z} \frac{\partial}{\partial z} \\
& -4 R^{2} \frac{1}{r} \frac{\partial \bar{c}}{\partial r} \frac{\partial \bar{c}}{\partial z} \frac{\partial^{2}}{\partial r \partial z}+2 R \frac{1}{r^{2}} \frac{\partial^{2} \bar{c}}{\partial r \partial z} \frac{\partial}{\partial z}-4 R \frac{1}{r} \frac{\partial^{2} \bar{c}}{\partial r \partial z} \frac{\partial^{2}}{\partial r \partial z}, \\
\boldsymbol{M}_{\mathbf{5}}= & -2 R^{2} \frac{\partial \bar{c}}{\partial z} \frac{\partial \bar{q}_{z}}{\partial r} \frac{\partial}{\partial z}-R \frac{\partial \bar{q}_{z}}{\partial r} \frac{\partial^{2}}{\partial z^{2}}+2 R^{2} \frac{\partial \bar{c}}{\partial r} \frac{\partial \bar{q}_{z}}{\partial r} \frac{\partial}{\partial r}+R \frac{\partial \bar{q}_{z}}{\partial r} \frac{\partial^{2}}{\partial r^{2}} \\
& -4 R^{2} \frac{1}{r} \frac{\partial \bar{c}}{\partial z} \bar{q}_{z} \frac{\partial}{\partial z}-2 R \frac{1}{r} \bar{q}_{z} \frac{\partial^{2}}{\partial z^{2}}+4 R^{2} \frac{1}{r} \frac{\partial \bar{c}}{\partial r} \bar{q}_{z} \frac{\partial}{\partial r}+2 R \frac{1}{r} \bar{q}_{z} \frac{\partial^{2}}{\partial r^{2}} \\
& -R \frac{1}{r} \frac{\partial \bar{q}_{z}}{\partial r} \frac{\partial}{\partial r}+4 R \frac{1}{r} \frac{\partial \bar{q}_{z}}{\partial r} \frac{\partial}{\partial r}-\mathrm{e}^{-R \bar{c}} R \frac{\partial \bar{c}}{\partial r}+\mathrm{e}^{-R \bar{c}} \frac{\partial}{\partial r}, \\
\boldsymbol{M}_{\mathbf{6}}= & -\frac{1}{r} \frac{\partial \bar{c}}{\partial z} \frac{\partial}{\partial r}+\frac{1}{r} \frac{\partial \bar{c}}{\partial r} \frac{\partial}{\partial z} .
\end{aligned}
$$


Here, $\hat{\psi}$ can be set to zero on all domain boundaries, while $\hat{\omega}$ and $\hat{c}$ vanish only at the far-field boundaries. The term $\hat{\omega}$ is set to $(1 / r)\left(\partial^{2} \hat{\psi} / \partial r^{2}\right)$ at the wall, and it vanishes on the axis. Both at the wall and on the axis, the normal derivative $\partial \hat{c} / \partial r$ is zero. A staggered grid is not required here, owing to the absence of the pressure variable and related singularities at the axis. Consequently, we can follow Vanaparthy et al. (2003) and use a highly accurate Chebyshev collocation method in the $z$-direction, for each of two subdomains that cover the regions $z \geqslant 0$ and $z \leqslant 0$, respectively. In this way, the grid points are concentrated around the interface. In the radial direction, a compact finite-difference scheme of third order at the wall and up to tenth-order in the interior is used (Lele 1992). Again, a Reynolds-number-like term with $R e=0.01$ is added to the momentum equation, in order to accelerate convergence. For the stability analysis on a domain with a length $l=10, N_{r}=30$ and $N_{z}=101$, the system matrix has a size of $3 \times N_{r} \times N z=9090 \times 9090$.

\section{Results for quiescent base state}

We begin by analysing the stability of the quiescent base state as a function of the viscosity ratio $R$. Before presenting the relevant dispersion relations, we discuss the influence of $R$ on the shape of the eigenfunctions.

\subsection{Shape of the eigenfunctions}

Figures 4 and 5 show the eigenfunction contours for pressure, all three velocity components, and concentration, for $R=1$ and $R=-1$, respectively. In contrast to the constant viscosity case, the eigenfunctions for $R \neq 0$ are not symmetric or skew symmetric with respect to $z=0$. Instead, for positive viscosity ratios $(R>0$, more viscous fluid on top) the maxima are shifted in the negative $z$-direction, while for negative viscosity ratios $(R<0$, more viscous fluid at the bottom) they are displaced in the positive $z$-direction. From the figures, it is apparent that the eigenfunctions for $R>0$ and $R<0$ are related as

$$
\begin{aligned}
\hat{p}_{R+}(r, z) & =-\hat{p}_{R-},(r,-z), \\
\hat{q}_{r, R+}(r, z) & =-\hat{q}_{r, R-},(r,-z), \\
\hat{q}_{\theta, R+}(r, z) & =-\hat{q}_{\theta, R-},(r,-z), \\
\hat{q}_{z, R+}(r, z) & =\hat{q}_{z, R-},(r,-z), \\
\hat{c}_{R+}(r, z) & =\hat{c}_{R-},(r,-z) .
\end{aligned}
$$

By substituting these relations into the governing equations (2.23)-(2.27) and keeping in mind that $\mathrm{e}_{R+}^{R \bar{c}(z)}=\mathrm{e}_{R-}^{R(\bar{c}(-z)-1)}, \bar{c}(z)=1-\bar{c}(-z),(\partial \hat{f} / \partial z)(+z)=-(\partial \hat{f} / \partial z)(-z)$, where $\hat{f}=\hat{p}, \hat{q}_{r}, \hat{q}_{\theta}, \hat{q}_{z}, \hat{c}$, and $(\partial \bar{c} / \partial z)(+z)=(\partial \bar{c} / \partial z)(-z)$, it is clear that for a quiescent base state, the systems of equations for $R+$ and $R-$ are equivalent, so that they yield the same eigenvalues. This is confirmed by the calculations, which yield the same growth rates for both cases. For this reason, we will focus mostly on positive viscosity ratios in the following.

By adding a positive or negative multiple of the concentration perturbation to the base concentration field, we are able to obtain a qualitative idea of the two possible perturbed interface shapes. For this purpose, $c=0.5$ contours are plotted in figure 6 for $\beta=1$ and $\beta=0$, where the solid (dashed) lines apply to positive (negative) values of $R$. For axisymmetric perturbations $(\beta=0)$, the heavier fluid moves down in the centre of the tube, while the lighter fluid rises in an annular region near the wall, or 

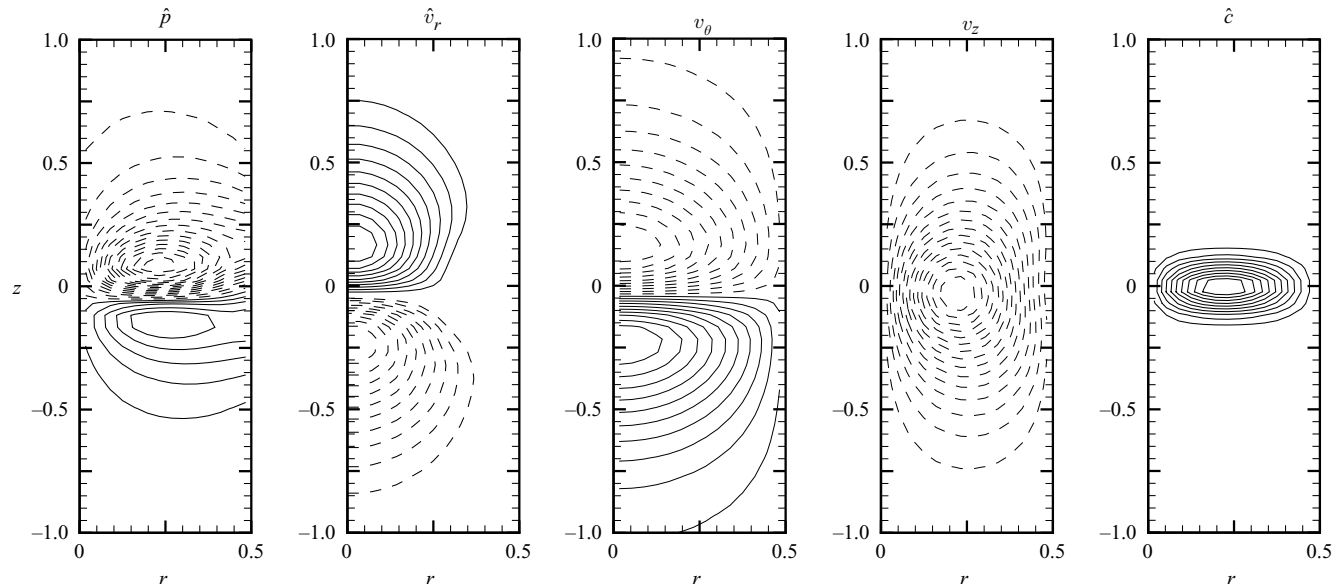

FIGURE 4 . Pressure $\hat{p}$, radial velocity $\hat{v}_{r}$, circumferential velocity $\hat{v}_{\theta}$, axial velocity $\hat{v}_{z}$ and the concentration eigenfunction $\hat{c}$ associated with the largest eigenvalue for $R=1, R a=10^{6}$ and $\delta=0.1$, for the azimuthal wavenumber $\beta=1$.
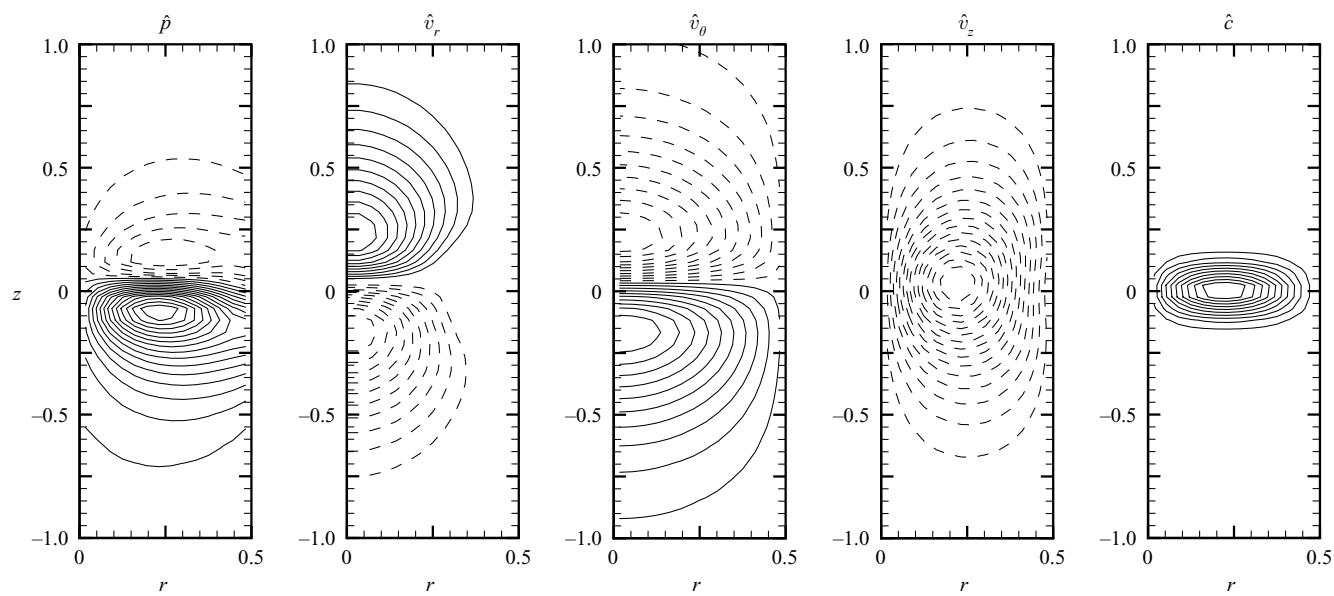

FIgURE 5 . Pressure $\hat{p}$, radial velocity $\hat{v}_{r}$, circumferential velocity $\hat{v}_{\theta}$, axial velocity $\hat{v}_{z}$ and the concentration eigenfunction $\hat{c}$ associated with the largest eigenvalue for $R=-1, R a=10^{6}$ and $\delta=0.1$, for the azimuthal wavenumber $\beta=1$.

vice versa. In the three-dimensional case $(\beta=1)$, the lighter fluid rises over one-half of the cross-section, while the heavier fluid sinks in the other half. This behaviour agrees with the interfacial shapes observed by Wilhelm \& Meiburg (2002) in nonlinear simulations for $R=2.3$ and $R a=1.147 \times 10^{6}$, and by Kuang et al. $(2003,2004)$ in experiments for $R=2.3$ and $R a=1.240 \times 10^{7}$, in the absence of net flow. The latter authors also show that the interface propagates more rapidly into the less viscous fluid than into the more viscous one, which is confirmed by figure 6 .

For large viscosity ratios $(R>3)$, the shape of the dominant perturbations changes qualitatively. For $R=1$, the eigenfunctions $\hat{v}_{r}$ and $\hat{v}_{z}$ had shown one vortex in the $(r, z)$-plane (figure 4). In contrast, for $R=5$, they exhibit two counter-rotating vortices (figure 7). Related to this is a change in the concentration eigenfunction at the wall. 

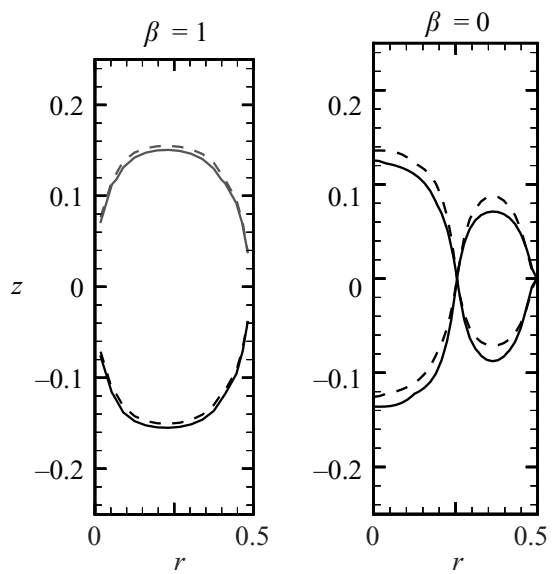

FIGURE 6. Qualitative shape of the interface for $\delta=0.1$ and $R a=10^{6}$, solid: $R=1$, dashed $R=-1$. For each value of $R$, there are two possible solutions.
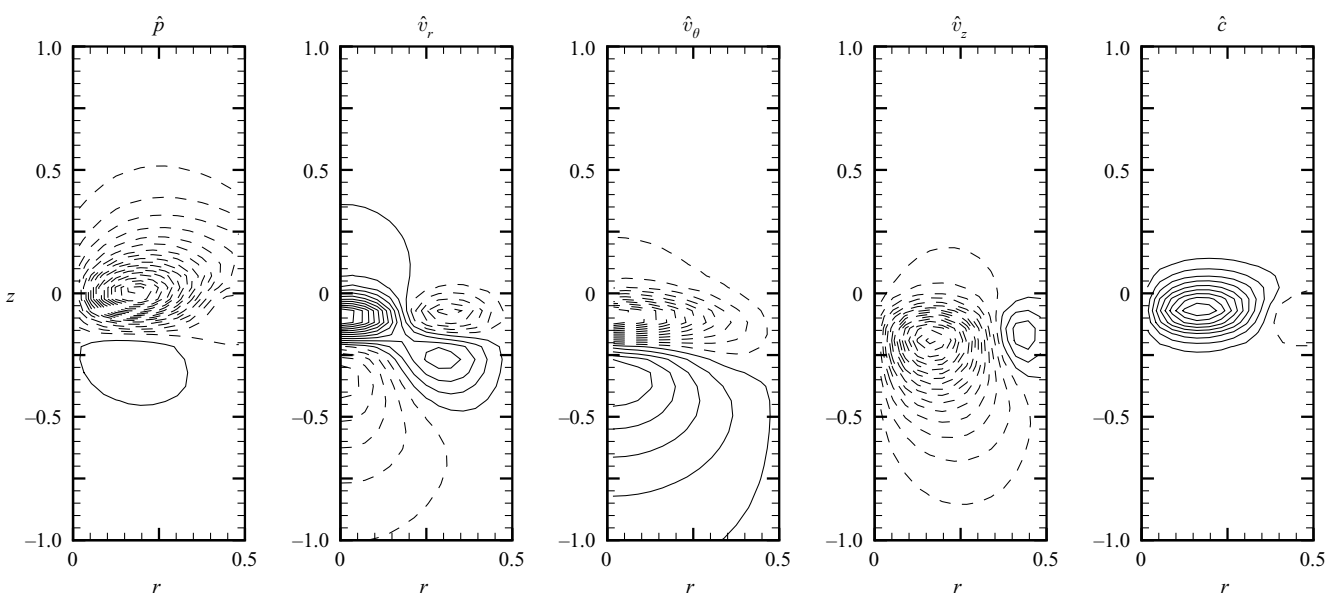

FiguRE 7. Pressure $\hat{p}$, radial velocity $\hat{v}_{r}$, circumferential velocity $\hat{v}_{\theta}$, axial velocity $\hat{v}_{z}$ and the concentration eigenfunction $\hat{c}$ associated with the largest eigenvalue for $R=5, R a=10^{6}$ and $\delta=0.1$, for the azimuthal wavenumber $\beta=1$.

A similar change is observed for the axisymmetric mode, where for large $R$ values, a region of counter-rotating vorticity appears near the axis (figure 8). This results in a dent at the tip of the downward moving finger as shown in figure 9 .

\subsection{Dispersion relations}

Figure 10 shows growth rates $\sigma$ as functions of the azimuthal wavenumber $\beta$, for the interface thickness $\delta=0.1, R a=10^{7}$, and various viscosity ratios. Note that, even though only integral values of $\beta$ are physically meaningful, the dispersion relations are drawn as continuous curves, in order to guide the eye. As expected, the growth rate decreases with increasing $R$ owing to the higher average viscosity of the fluid system, which has a damping effect. For small values of $R$, the dispersion relation has a global maximum for the three-dimensional mode $\beta=1$. The growth rates of the axisymmetric and three-dimensional modes are affected somewhat differently by larger viscosity ratios (figure 10). While both modes are exponentially damped 

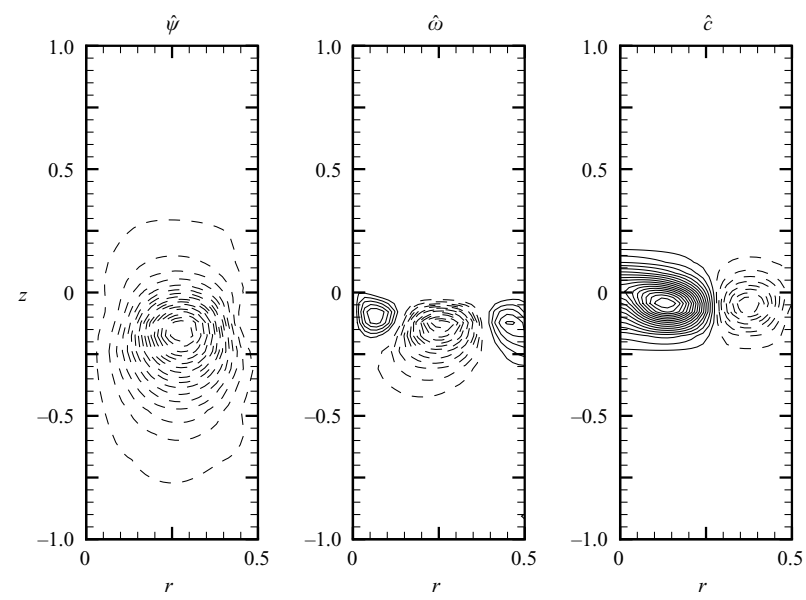

FIGURE 8 . Axisymmetric perturbations of the streamfunction $\hat{\psi}$, vorticity $\hat{\omega}$ and the concentration $\hat{c}$ associated with the largest eigenvalue for $R=4, R a=10^{6}$ at a characteristic interface thickness of $\delta=0.1$.
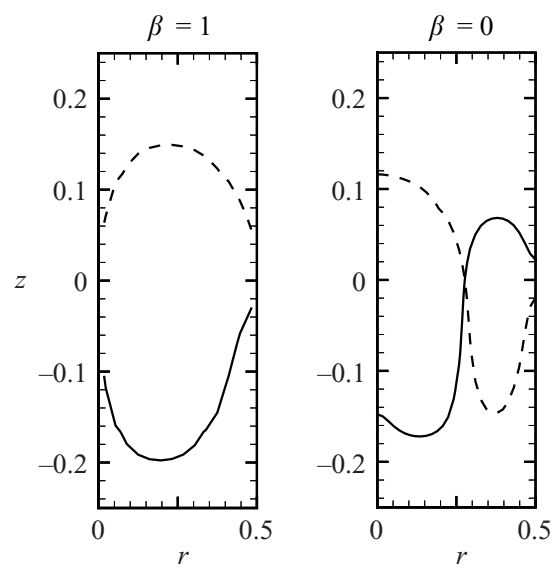

FIGURE 9. Qualitative shape of the interface for $\delta=0.1, R a=10^{6}$ and $R=5$. Each of the solid and the dashed lines visualize one of the two possible solutions provided by $\hat{c}$.

by increasing $R$, the damping constant is larger for the three-dimensional mode. Consequently, for $R>3.48$, the axisymmetric mode $(\beta=0)$ exhibits the higher growth rate. Note that for lower Rayleigh numbers, a comparable crossover of the curves for $\beta=0$ and $\beta=1$ is not observed, cf. figure 11 for $R a=10^{5}$.

Figure 12 indicates the dependence of the growth rates for $\beta=0$ and 1 on $R a$, for various viscosity ratios. For all $R$, an increase of $R a$ is seen to result in higher growth rates, until a plateau is reached for $R a>10^{6}$. From these curves, we can identify a critical value $R a_{\text {crit }}$ for each viscosity ratio, below which the base state is stable to perturbations. Figure 13 depicts $R a_{\text {crit }}$ as a function of the viscosity ratio for the interface thicknesses $\delta=0.1$ and 0.5 . For small viscosity ratios $R \leqslant 2$, the thinner interface exhibits a lower $R a_{\text {crit }}$, so that it is more unstable. However, for viscosity ratios $R \geqslant 3$ the thicker interface is more unstable. This behaviour is confirmed by figure 14, which shows that at $R=5$ the growth rates for $\beta=0$ and 1 have a maximum 

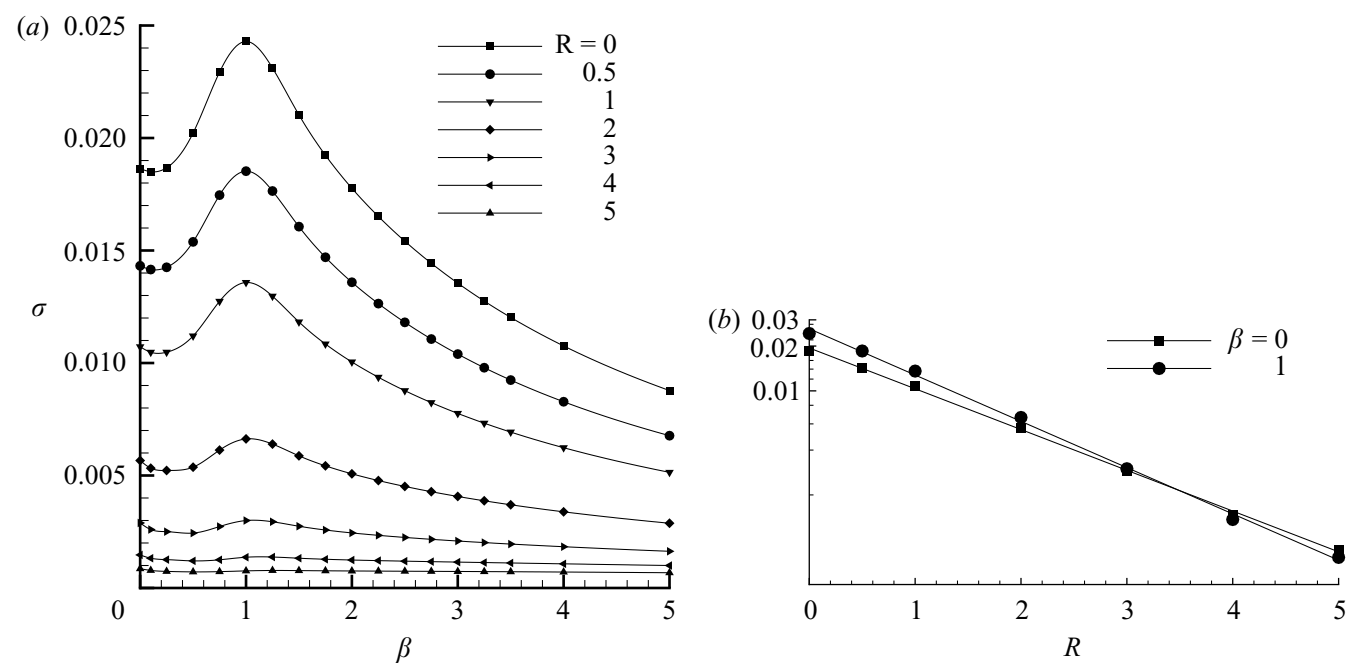

FIGURE 10. Dispersion relationships for $\delta=0.1, R a=10^{7}$ and various $R$ values. In $(b)$, the growth rate of the axisymmetric mode $(\beta=0)$ is compared with that of the most amplified three-dimensional mode $\beta=1$. For values of $R$ below 3.48, the three-dimensional mode is seen to dominate, whereas the axisymmetric mode has a higher amplification rate above this value.
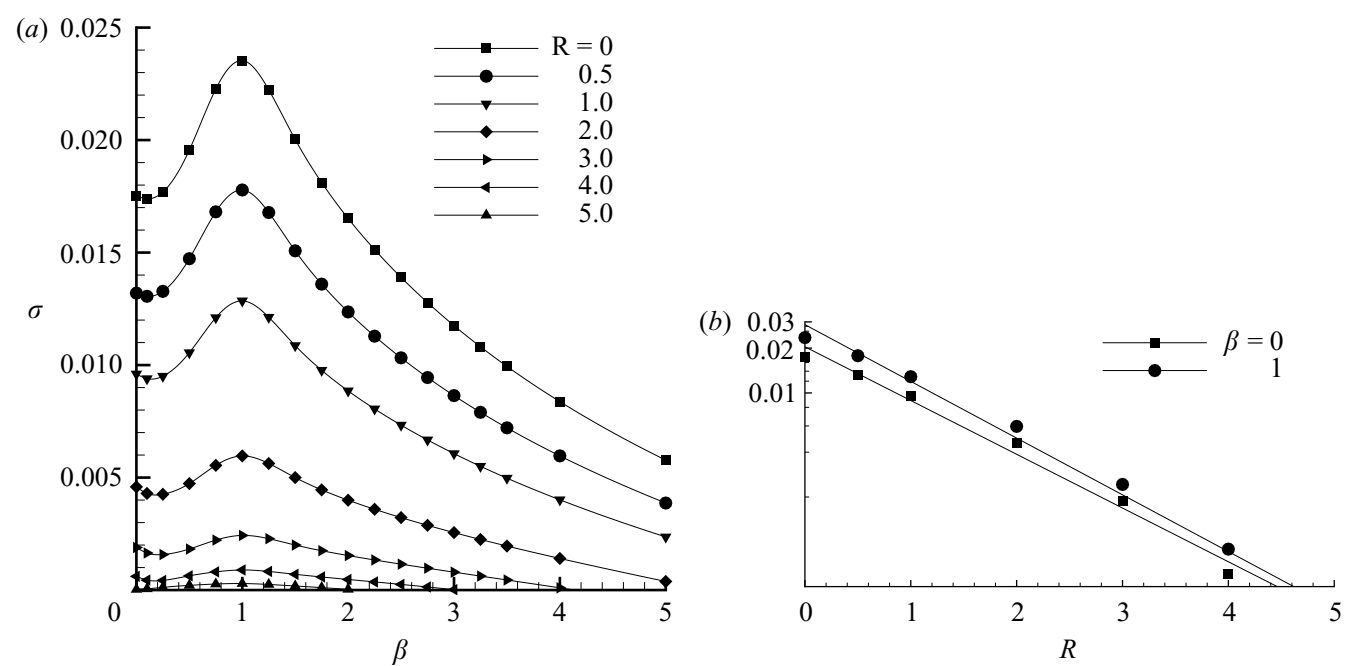

FIGURE 11. Dispersion relationships for $\delta=0.1, R a=10^{5}$ and various $R$. In $(b)$, the corresponding damping behaviour of the $\beta=0$ and $\beta=1$ modes is shown. At this lower $R a$ value, the crossover observed for $R a=10^{7}$ does not occur.

near $\delta=0.5$. For $R=0$, on the other hand, the growth rates decrease monotonically with increasing $\delta$.

While this finding may be somewhat counterintuitive, Goyal \& Meiburg (2004) had made a similar observation for the corresponding situation in a Hele-Shaw cell, cf. also the experimental investigation of Fernandez et al. (2002). Those authors attributed this behaviour to the fact that for thick interfaces and large viscosity ratios, the eigenfunction maximum shifts a considerable distance into the less viscous fluid, so that the perturbation can grow in a lower-viscosity environment. For moderate 


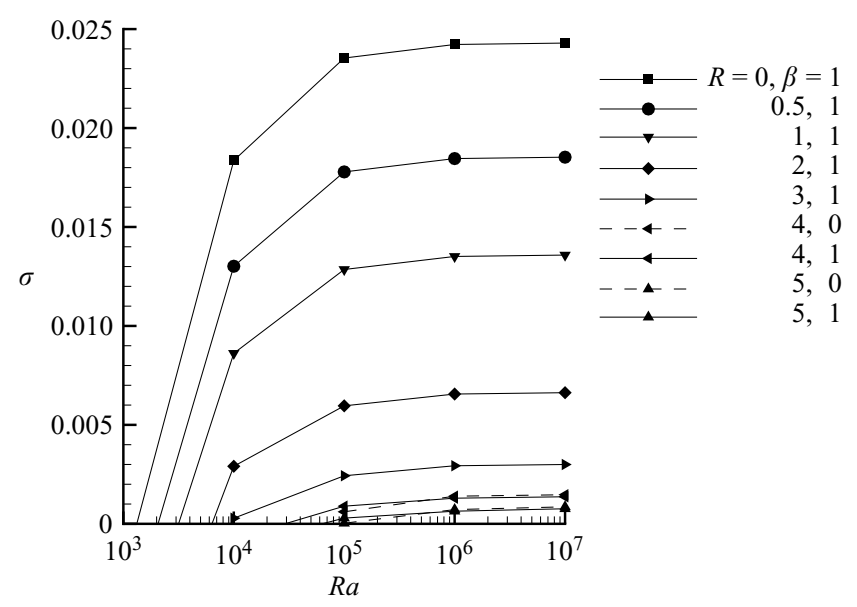

FIGURE 12. Growth rates $\sigma$ of the axisymmetric and leading azimuthal modes as functions of $R a$, for various viscosity ratios. Above $R a \approx 10^{6}$, a plateau is reached.

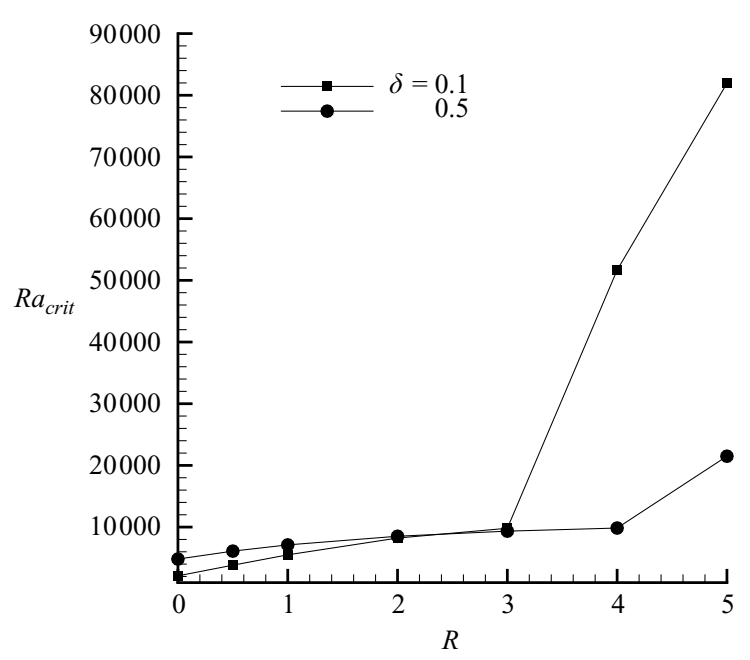

FIGURE 13. The critical Rayleigh number $R a_{c r i t}$ as a function of $R$. For large-viscosity ratios, the thicker interface becomes unstable at smaller Rayleigh numbers than the thinner one.

values of $\delta$, this effect can offset the stabilizing influence of an increasing interface thickness. For thin interfaces, on the other hand, the eigenfunction is forced to extend significantly into the more viscous fluid, which dampens its growth. Figure 15 demonstrates that a similar shift into the less viscous environment occurs for the present case of a capillary tube. Table 2 provides the $z$-location of the eigenfunction maximum, the corresponding base concentration value $\bar{c}$, the local viscosity $\mu$, and the concentration gradient $\partial \bar{c} / \partial z$. It shows that for $\delta=0.5$, the eigenfunction maximum is located in the least viscous environment. Since the destabilizing base concentration gradient is still reasonably strong, this $\delta$ value leads to the highest overall growth rate.

Figure 16 provides more complete information for the intermediate interface thickness $\delta=0.5$. Note that even for $R=5$, the global maximum of the dispersion relation is located at $\beta=1$. Hence, for the present interface thickness, a crossover 


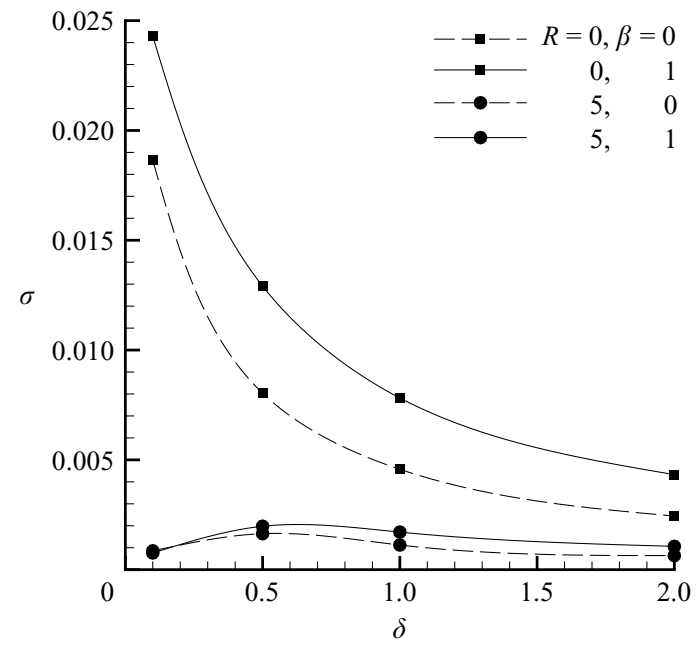

FiguRE 14. Growth rates for $\beta=0$ and 1 as functions of the interfacial thickness $\delta$, for $R a=10^{7}$. For $R=5$, the growth rates display a maximum near $\delta=0.5$, whereas for $R=0$ they decrease monotonically.
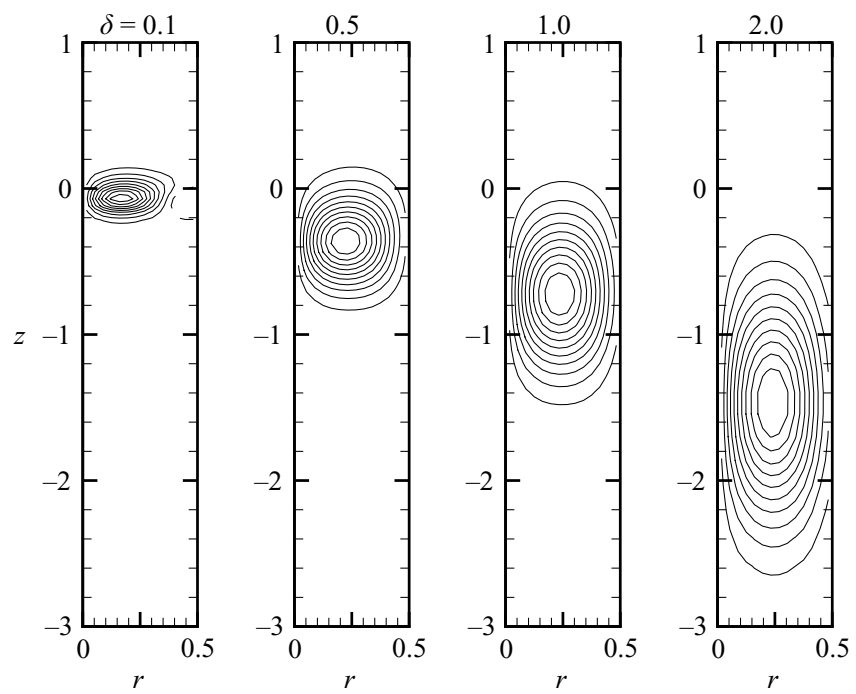

FIGURE 15. $R=5, R a=10^{6}, \beta=1$ : For larger interface thicknesses, the maximum of the concentration eigenfunction $\hat{c}$ is increasingly shifted into the less viscous fluid.

with the $\beta=0$ mode does not occur, so that the dominant mode is three-dimensional in nature for all viscosity ratios.

\section{Influence of a net axial base flow}

In their experimental investigation, Kuang et al. (2004) observed that a small amount of net axial flow can stabilize the azimuthal instability mode, so that the axisymmetric mode becomes dominant. A minimum flow rate to accomplish this is not established, however. This finding could not be explained on the basis of the constant viscosity linear stability results by Vanaparthy et al. (2003). In the following, 


$\begin{array}{lllll}\delta & 0.1 & 0.5 & 1.0 & 2.0 \\ z & -0.07 & -0.38 & -0.71 & -1.49 \\ \bar{c} & 0.1898 & 0.1421 & 0.1578 & 0.1461 \\ \mu & 2.583 & 2.035 & 2.201 & 2.076 \\ \frac{\partial \bar{c}}{\partial z} & 3.456 & 0.633 & 0.341 & 0.162 \\ \sigma & 0.76 \times 10^{-3} & 0.19 \times 10^{-2} & 0.17 \times 10^{-2} & 0.10 \times 10^{-2}\end{array}$

TABle 2. $R=5, R a=10^{6}, \beta=1$. Position $z$, base concentration $\bar{c}$, viscosity $\mu$ and base concentration gradient $\partial \bar{c} / \partial z$ at the location of the concentration eigenfunction maximum. The most unstable combination of low-viscosity environment and strong base concentration gradient leads to a maximum growth rate $\sigma$ for the intermediate interface thickness $\delta=0.5$.
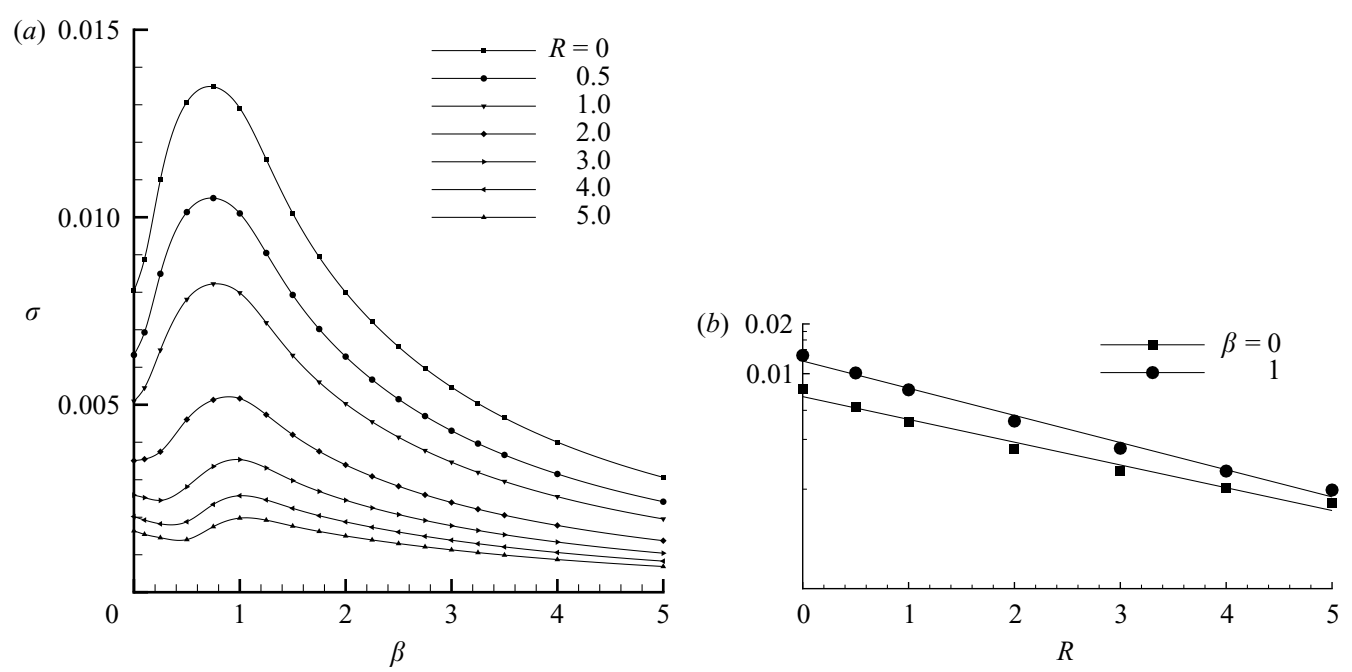

FIGURE 16. Dispersion relationships for $\delta=0.5, R a=10^{7}$ and various $R$. In $(b)$, the corresponding damping behaviour of the $\beta=0$ and $\beta=1$ modes is shown. For this interface thickness, a crossover is not observed, so that the dominant mode remains three-dimensional in nature for all viscosity ratios.

we will try to evaluate the influence of viscosity variations on this competition between axisymmetric and azimuthal modes. The influence of a net flux is also of interest in the context of the finger shape in miscible displacements in capillary tubes, cf. the joint experimental and computational investigations by Petitjeans \& Maxworthy (1996) and Chen \& Meiburg (1996).

Based on the work by Taylor (1953), Vanaparthy et al. (2003) introduced a framework for analysing the effect of a small net axial velocity on the linear stability of the miscible interface considered above. For a Poiseuille flow profile of the form

$$
\bar{q}_{z}=u_{\max }\left(1-4 \frac{r^{2}}{d^{2}}\right)
$$

Taylor had shown that radial flow-induced concentration variations are rendered small by the action of molecular diffusion, provided that

$$
P e=\frac{u_{\max } d}{D}=R a \frac{u_{\max }}{U^{*}}=R a u_{n} \ll 7.6^{2} \frac{\delta}{d} .
$$




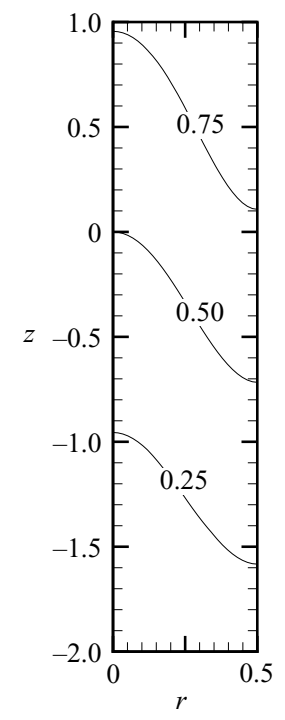

FIGURE 17. Influence of the net axial flow on the base concentration profile. Shown are the contours $\bar{c}=0.25,0.5$ and 0.75 for a net upward flow with $P e=50$ and $\delta=2$.

Under these conditions, he finds that the concentration profile of the passive scalar is given by

$$
c(r, \xi)=\frac{1}{2}+\frac{1}{2} \operatorname{erf}\left(\frac{\xi}{2 \sqrt{k t}}\right)+\frac{P e}{16 \sqrt{\pi k t}}\left(r^{2}-2 r^{4}\right) \exp \left(-\frac{\xi^{2}}{4 k t}\right) .
$$

Here, $\xi=z-u_{\max } t / 2$ denotes the axial coordinate in a reference frame moving with the mean velocity of the flow, while $k=u_{\text {max }}^{2} d^{2} / 768 D$ represents the well-known Taylor dispersion coefficient. Figure 17 depicts the contours $c=0.25,0.5$ and 0.75 of this base concentration profile. In order to investigate the influence of this Poiseuille flow on the interfacial instability, we set

$$
\delta=2 \sqrt{k t}
$$

and assume a quasi-steady state $\delta=$ const. While proceeding analogously to the noflow case, we must keep in mind that the current situation, in which both density and viscosity depend on the concentration, is clearly distinct from the case of a passive scalar analysed by Taylor (1953). As a result, the real flow will not be exactly of Poseuille type. Nevertheless, as long as radial concentration variations remain small, the Poiseuille flow assumption should represent a good approximation.

The derivation of the generalized eigenvalue problems in $\$ \S 2.2$ and 2.3 was performed for the general case of a base concentration profile that varies in $z$ and $r$, and a base flow with a component in the $z$-direction. Test calculations show that even for small values of $u_{n}$, long domains of $l=25$ and fine resolutions of $N_{z}=121$ and $N_{r}=19$ are necessary to obtain converged results. In order to avoid numerical stability problems near the in- and outflow boundaries, we furthermore had to gradually reduce the base flow near these.

\subsection{Results}

Because of condition (4.2), the above approach is restricted to a small area of the $\delta$ $R a-u_{n}$ parameter space. Therefore, in order to reach sufficiently large Péclet numbers, only relatively thick interfaces with $\delta=2$ are considered. 

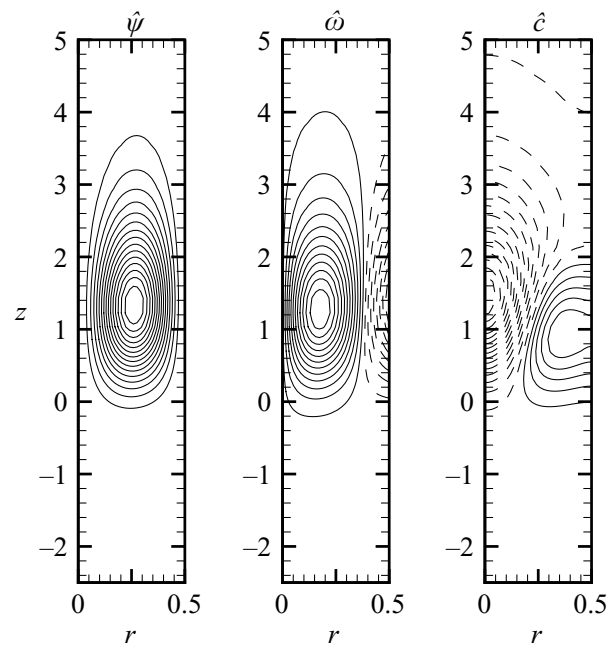

FIGURE 18. Axisymmetric perturbations of the streamfunction $\hat{\psi}$, vorticity $\hat{\omega}$ and the concentration $\hat{c}$ associated with $R=1, R a=10^{5}, \delta=2.0$ for a base flow $u_{n}=0.0005$. Notice the shift in the direction of the base flow, i.e. towards the more viscous fluid.
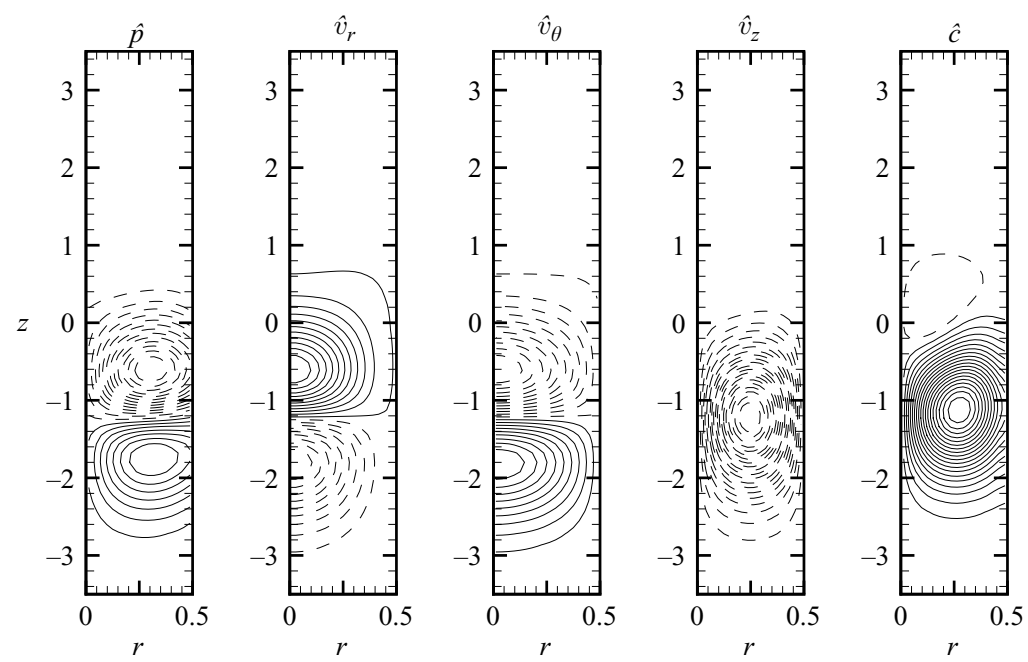

FIGURE 19 . Pressure $\hat{p}$, radial velocity $\hat{v}_{r}$, circumferential velocity $\hat{v}_{\theta}$, axial velocity $\hat{v}_{z}$ and the concentration eigenfunction $\hat{c}$ associated with the largest eigenvalue for $u_{n}=0.0005, R=1$, $R a=10^{5}$ at a circumferential wavenumber $\beta=1$. Notice the shift towards the less viscous fluid, i.e. against the base flow.

Figure 18 depicts the eigenfunctions of the axisymmetric instability mode for $R=1$, $R a=10^{5}$ and $u_{n}=0.0005$. Compared to the no-flow case, the eigenfunctions are shifted in the flow direction, i.e. towards the more viscous fluid. As can be seen clearly from the concentration eigenfunction, this shift is more pronounced near the axis, where the flow velocity is larger, than near the wall. Figure 19 shows the corresponding results for the three-dimensional mode $\beta=1$. In contrast to the axisymmetric mode, the three-dimensional eigenfunctions display a shift towards the less viscous fluid, i.e. against the flow direction. The reason for this qualitatively different behaviour of the $\beta=0$ and $\beta=1$ modes is not entirely clear. 


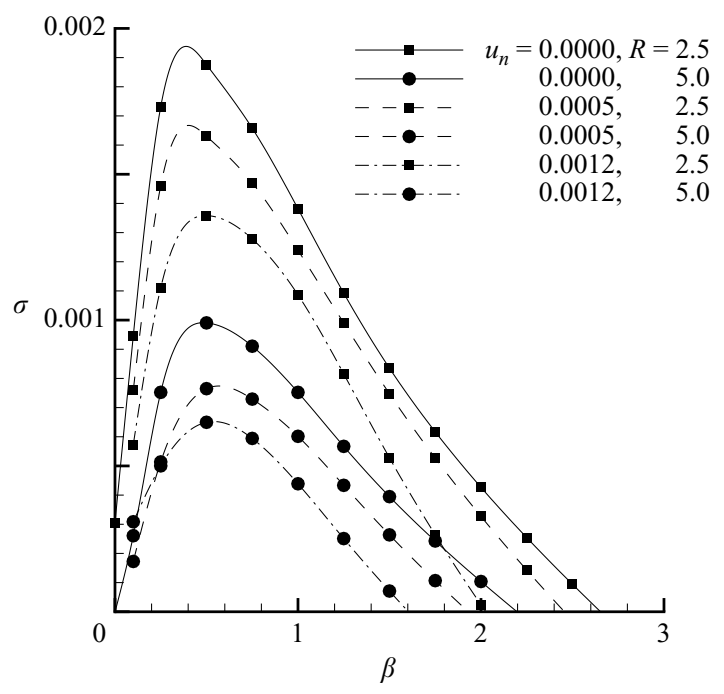

FiguRE 20. Growth rate $\sigma$ as a function of the wave length $\beta$ for $R=2.5$ and 5 , and different centreline velocities $u_{n}$. $R a=10^{5}$. A crossover takes place for $R=5$ and small wavenumbers, which suggests that for large-viscosity contrasts the axisymmetric mode is destabilized by the net flow, while the azimuthal mode is stabilized, in line with the experimental observations by Kuang et al. (2004).

In the presence of a base flow, the system of linear governing equations displays a symmetry for $\left(R+, u_{n}+\right)$ and $\left(R-, u_{n}-\right)$. This confirms our expectation that the situation of a more viscous fluid above a less viscous one with an upward net flow gives the same growth rates as a less viscous fluid above a more viscous one with a downward net flow.

Figure 20 depicts dispersion relations for velocities $u_{n}=0,0.0005$ and 0.0012 , with $R=2.5$ and 5 , at $R a=10^{5}$. The graphs indicate that the $\beta=1$ mode dominates for the whole parameter spectrum considered. Note, however, the crossover of the $R=5$ curves near $\beta=0$, which indicates a destabilizing influence of the axial net flow on the axisymmetric mode. This is in contrast to the $\beta=1$ mode, which is stabilized by the net flow. This might indicate that, for sufficiently high flow rates and viscosity ratios, the axisymmetric mode may become more unstable than the $\beta=1$ mode, in line with the experimental observations of Kuang et al. (2004). Those authors state that a minimum net flow was required to obtain an axisymmetric finger; however, they did not measure the minimum required velocity, so that it is not possible to make a more quantitative comparison. We were unable to obtain converged results for flow rates larger than $u_{n}=0.0012$, for which there may be several potential reasons. Based on extensive testing, we think it is unlikely that this is due to the computational resolution. Rather, we believe that convergence may no longer be achieved because the base state (4.3) represents a less and less valid approximation of the governing equations as $u_{n}$ increases. Apart from the presence of density and viscosity variations, which did not exist in Taylor's analysis, $u_{n}$ must satisfy condition (4.2). Hence, for $R a=10^{5}, u_{n}=$ 0.0012 and $\delta / d=2$, we are already at the very limit of validity of this approximation.

\section{Conclusion}

We have analysed the influence of a concentration-dependent viscosity on the density-driven instability between miscible fluids in a vertically oriented capillary 
tube. The main focus of the investigation, which extends the constant viscosity study by Vanaparthy et al. (2003), is to establish the dominant instability mode as a function of Rayleigh number, viscosity ratio and interfacial thickness parameter. Of particular interest is the competition between the axisymmetric and first azimuthal modes, for which the experiments of Kuang et al. (2003) demonstrate a very sensitive balance. Towards this end, we formulated a generalized eigenvalue problem, based on the three-dimensional Stokes equations, whose solution provides the dispersion relations and eigenmodes as functions of the governing dimensionless parameters.

In the absence of a net flow, the system of linear equations demonstrates certain symmetry properties, which indicate that the growth rates do not depend on which of the two fluids is the more viscous one. For most parameter combinations, the azimuthal mode $\beta=1$ is found to have larger growth rates than the axisymmetric mode. However, for thin interfaces and large Rayleigh numbers, the axisymmetric mode dominates above a certain viscosity ratio. Furthermore, the interface is seen to propagate more rapidly into the less viscous fluid.

An unexpected result is found regarding the influence of the interface thickness on the instability. For large viscosity ratios, intermediate interface thicknesses are found to be more unstable than either very thin or very thick interfaces. The reason for this behaviour is traced to a shift of the eigenfunctions towards the less viscous fluid. This shift allows the instability to grow in an overall less viscous environment. In this context, it is important to appreciate that the vertical extent of the eigenfunction depends on both the interfacial thickness and the tube radius. For very thin interfaces, this forces the eigenfunction to extend deep into the more viscous fluid, which lowers the growth rate. On the other hand, for very thick interfaces the growth rate decreases because the destabilizing density gradient is weakened. For intermediate interface thicknesses, an optimal combination of destabilizing density gradient and shift into a less viscous environment is achieved. This observation has a counterpart for HeleShaw problems, as pointed out by Goyal (2003).

In the presence of a net axial flow, the governing equations exhibit a symmetry regarding $\left(R+, u_{n}+\right)$ and $\left(R-, u_{n}-\right)$ combinations. This indicates that the upward flow of a more viscous fluid above a less viscous one gives rise to the same growth rate as the downward flow of a less viscous fluid above a more viscous one. For large viscosity ratios, the axisymmetric mode becomes destabilized by increasing net flows, whereas the leading azimuthal mode is stabilized. While we were unable to obtain converged results at flow rates that are sufficiently large for the axisymmetric mode to dominate, the trend of the results nevertheless is in line with the experimental observations of Kuang et al. (2004). A more quantitative comparison is not possible at this time, since neither the experimental data of Kuang et al. (2004) nor the present linear stability results provide a value for the minimum net flux required for the stabilization of the azimuthal mode.

Throughout the investigation, we employed a constant diffusion coefficient. This assumption is common in linear stability investigations of variable viscosity, miscible fluids. Nevertheless, its validity is somewhat questionable in light of the StokesEinstein relation, which predicts that the product of viscosity and diffusivity remains approximately constant (see e.g. Probstein 1994). Efforts towards quantifying the influence of this assumption are currently underway.

Support for this work was received from the NASA Microgravity and NSF/ITR programs, as well as from the Department of Energy and the UC Energy Institute, 
and through an NSF equipment grant. M. P. thanks the Ernest-Solvay Stiftung for support during his stay at UCSB.

\section{REFERENCES}

Chen, C.-Y. \& Meiburg, E. 1996 Miscible displacements in capillary tubes. Part 2. Numerical simulations. J. Fluid Mech. 326, 57.

Fernandez, J., Kurowski, P., Petitjeans, P. \& Meiburg, E. 2002 Density-driven, unstable flows of miscible fluids in a Hele-Shaw cell. J. Fluid Mech. 451, 239.

Fletcher, C. A. J. 1991 Computational Techniques for Fluid Dynamics, Springer.

Goyal, N. \& Meiburg, E. 2004 Gravitational instabilities of miscible fluids with variable density and viscosity in a Hele-Shaw cell. J. Fluid Mech. 516, 211.

Graf, F., Meiburg, E. \& Härtel, C. 2002 Density-driven instabilities of miscible fluids in a HeleShaw cell: linear stability analysis of the three-dimensional Stokes equations. J. Fluid Mech. 451, 261.

Kuang, J., Maxworthy, T. \& Petitjeans, P. 2003 Miscible displacements between silicone oils in capillary tubes. Eur. J. Mech. 22, 271.

Kuang, J., Maxworthy, T. \& Petitjeans, P. 2004 Velocity fields and streamline patterns of miscible displacements in cylindrical tubes. Exps. Fluids 37, 301.

Lele, S. K. 1992 Compact finite difference schemes with spectral-like resolution. J. Comput. Phys. $103,16$.

MaschHoff, M. J. \& SoRensen, D. C. 1996 P-ARPACK: an efficient portable large scale eigenvalue package for distributed memory parallel architectures. Applied Parallel Computing in Industrial Problems and Optimization. Lecture Notes in Computer Science 1184. Springer.

Petitjeans, P. \& Maxworthy, T. 1996 Miscible displacements in capillary tubes. Part 1. Experiments. J. Fluid Mech. 326, 37.

Probstein, R. F. 1994 Physicochemical Hydrodynamics: An Introduction. John Wiley.

Sorensen, D. C. 1992 Implicit application of polynomial filters in a k-step Arnoldi method. SIAM J. Matrix Anal. Appl. 13, 357.

TAYLOR, G. I. 1953 Dispersion of soluble matter in solvent flowing slowly through a tube. Proc. R. Soc. Lond. A 219, 186.

Vanaparthy, S. H., Meiburg, E. \& Wilhelm, D. 2003 Density-driven instabilities of miscible fluids in a capillary tube: linear stability analysis. J. Fluid Mech. 497, 99.

VerzicCO, R. \& ORLANDI, P. A. 1996 A finite-difference scheme for three-dimensional incompressible flows in cylinder coordinates. J. Comput. Phys 123, 402.

Wilhelm, D. \& Meiburg, E. 2004 Three-dimensional spectral element simulations of variable density and viscosity, miscible displacements in a capillary tube. Comput. Fluids 33, 485. 